\title{
Covid-19 and Diabetes Mellitus: What do you know?
}

\author{
Donizete Tavares da Silva, BS ${ }^{1}$, César Ernani Vasconcelos Rufino, BS $^{\mathbf{1}}$, Nayra Danielly dos \\ Santos Marques, $\mathbf{B S}^{2}$, Rayanne Timóteo de Sousa Flizikowski, $\mathbf{B S}^{2}$, André Gonçalves da Silva, \\ $\mathrm{MD}^{3}$, Wallace Rodrigues de Holanda Miranda, $\mathrm{MD}, \mathrm{PhD}^{3^{*}}$ \\ ${ }^{I}$ College of Medicine, Federal University of Piauí, Teresina, Piaui, Brazil \\ ${ }^{2}$ College of Medicine, Uninovafapi University Center, Teresina, Piaui, Brazil \\ ${ }^{3}$ General Medical Department, University Hospital of the Federal University of Piauí, Teresina, Piaui, Brazil
}

*Corresponding Author: Wallace Rodrigues de Holanda Miranda, MD, PhD, General Medical Department, University Hospital of the Federal University of Piauí, Teresina, Piaui, Brazil. Email:wallacerhmiranda@yahoo.com.br

\begin{abstract}
The COVID-19 pandemic caused by Severe acute respiratory syndrome coronavirus 2 (SARS-Cov-2) has become a global public health emergency. Diabetes Mellitus (DM), in turn, is a slowly evolving pandemic. Due to the crossing of these two pandemics, it is important to discuss the impact of COVID-19 on this population. We describe the aspects involved in SARS-Cov-2 infection in diabetic patients. We present the pathophysiology, interference of diabetes medications, prognosis of diabetic patients with COVID-19 and the management of these patients. The endocrine associations between diabetes mellitus and COVID-19 demonstrate an important relationship in their pathophysiology. DM is one of the risk factors for the development of unfavorable results.
\end{abstract}

Abbreviations: ACE-2:Angiotensin-Converting Enzyme 2; ARDS: Acute Respiratory Distress Syndrome; ARB: Angiotensin Receptor Blockers; COVID-19: Coronavirus Disease 2019; DM: Diabetes Mellitus; DPP4: Dipeptidyl peptidase 4; GCSF: Granulocyte Colony Stimulating Factor; GLP-1: Glucagon-Like Peptide-1 agonist; ICUs: Intensive Care Units; IDPP4: Inhibitors of dipeptidyl peptidase 4; IFN $\gamma$ : Interferon- $\gamma$; IL-1 $\beta$ : Interleucina-1 $\beta$; IL- 4: Interleucina-4; IL-6: Interleucina- 6; IL- 8: Interleucina-8; IL10: Interleucina-10; IP-10: Protein 10 Induced by Gamma Interferon (IP-10) MCP-1: Monocyte Chemoprotein protein 1; MERS: Middle East respiratory syndrome; MIP-1A: Macrophage Inflammatory Protein-1A (MIP-1A); NLRP3:Nucleotide-binding oligomerization domain, the 3-pirin domain NK: Natural Killer Cells (NK); SARS-CoV-2: Severe acute respiratory syndrome coronavirus 2; SGLT-2: Sodium Glucose-2 Cotransporter (SGLT-2); TNF- $\alpha$ : Tumor Necrosis Factor- $\alpha$ (TNF- $\alpha$ )

Keywords: COVID-19, Diabetes Mellitus, Diabetes Medications, SARS-CoV-2.

\section{BACKGROUND}

The COVID-19 pandemic caused by Severe acute respiratory syndrome coronavirus 2 (SARS-Cov-2) has become a global public health emergency. Most patients develop mild to moderate flu syndrome, however other patients, especially those with comorbidities, may develop severe pneumonia requiring mechanical ventilation and presenting a high risk of mortality. In addition, the pandemic represents a challenge for the continuity of care and the prevention of risks related to pre-existing chronic diseases. Diabetes, on the other hand, is a slowly evolving pandemic, being one of the main causes of death in the world in 2019.
Currently, it is estimated that 425 million people live with the disease [1]. With the crossing of these two pandemics, it is important to discuss the impact of COVID-19 on this population.

We describe the aspects involved in SARS-Cov2 infection in diabetic patients. We investigated the pathophysiology, interference of diabetes medications, prognosis of diabetic patients with COVID-19 and the management of these patients.

\section{Methodology}

This is a bibliographic search conducted in August 2020. The literature review was carried out in the Virtual Health Library (VHL), 
SCIELO and PubMed database. The research used keywords: "COVID-19", "SARS-CoV-2" and "diabetes mellitus". The filters were: year of publication, type of article and text availability. Inclusion Criteria: papers published from 2020 onwards, case reports, meta-analyzes, literature review, controlled and randomized testing, clinical trials, systematic review, editorials, technical notes, expert opinion, texts available in full, and adaptation to study theme. Exclusion criteria: articles that are not complete electronically and are not available in Portuguese, English or Spanish.

77 articles were selected from 148 obtained. There are 55 literature reviews, 13 systematic reviews and Meta-analyzes, 8 case reports, 4 editorials, 2 retrospective cohort studies, 2 original articles, 2 technical notes and 1 expert opinion.

\subsection{Pathophysiology}

Of the selected articles, 2 report that the pathophysiology of SARS-COV-2 infection is not yet fully understood, requiring further studies for such clarification. In view of this, 13 articles already point out that the AngiotensinConverting Enzyme 2 (ACE2) is a gateway for the virus in the host cell, and 11 of these suggest that the diabetic patient has an increase in the expression of this enzyme in lung tissues.

Among the factors related to diabetes, 13 articles report an increase in inflammatory mediators and coagulation factors, and 11 articles explain the immune deficiency present in these patients. In addition, 7 articles state that hyperglycemia and insulin resistance are exacerbated during COVID-19. Thus, the endocrine associations between diabetes and COVID-19 demonstrate an important relationship in the pathophysiology between both.

Contagion by SARS-COV-2 occurs mainly through respiratory drains that are expelled from an infected individual or through direct and indirect contact of the mucous membranes with objects infested with the virus [2]. After contagion, the virus seeks host cells through ACE-2, found mainly in the cardiovascular system, kidneys, lungs and brain [3]. Some factors mediate this entry, such as the serine transmembrane protease 2 (TMPRSS2) responsible for activating the spike (S) protein that is released by cleavage by the furin protease, enabling endosomal cell invasion [4]. Studies suggest that the expression of both ACE-2 and furin is increased in diabetic patients, possibly related to the glycosylation of the enzyme induced by uncontrolled hyperglycemia. Therefore, diabetic patients are likely to be infected and have a clinical course of the disease. However, there is no consensus on the relationship between diabetes and contagion [5].

The clinical course of COVID-19 goes through 3 stages: stage I involves early viral infection with constitutional symptoms, stage II involves Acute Respiratory Distress Syndrome (ARDS) and hypoxia, which result from direct viral cytotoxicity of the lung due to inflammatory activation, and stage III, which is a state severe with organ dysfunction and cytokine storm with immune dysfunction[2]. The disease progresses severely as soon as it moves to stage II and the following. Thus, the association between diabetes and the worsening of COVID-19 should be analyzed in accordance with the factors prevalent in these states.

Diabetes in its pathophysiology has a chronic low-intensity pro-inflammatory fator[6]. Studies infer the presence of increased cytokines, such as interleukin-6 (IL-6) and interleukin-8 (IL-8), C-reactive protein and ferritin in diabetic patients compared to the group without diabetes [7]. In addition, increased oxidative stress[6] and late hyperinflammatory reaction are regular findings of hyperglycemia. Thus, it is concluded that there is an inability in the physiological control of inflammatory mediators in these patients [8].

In addition to the factors inherent in diabetes, SARS-CoV-2 leads to another increase in the release of pro-inflammatory cytokines and chemokines, including IL-1 $\beta$, IL-4 and IL-10 interleukins, monocyte chemoprotein protein 1 (MCP- 1), interferon- $\gamma$ (IFN $\gamma$ ), protein 10 induced by gamma interferon (IP-10), granulocyte colony stimulating factor (GCSF), macrophage inflammatory protein-1 A (MIP-1A) and tumor necrosis factor- $\alpha$ (TNF- $\alpha$ ), inducing the picture for a potential "cytokine storm". Thus, if the two diseases are accompanied by an increase in inflammatory mediators and physiological control is already inefficient, the inflammatory response will be exacerbated, causing the patient to worsen clinically [9].

Another important factor is that diabetes has a high prevalence in the elderly, obese or with cardiovascular problems, such as an increased risk of cardiac arrhythmias [10], which in itself could help explain the relationship with worsening of COVID-19 [11]. In this sense, the vascular system is affected with endothelial 
dysfunction and increased platelet aggregation, inducing a hypercoagulable prothrombotic condition [12] and some markers such as fibrinogen and D-dimer can be found in patients diabetics [4]. The diabetic's lung is greatly affected by this vascular factor with hyperglycemia and oxidative stress, causing micro and macroangiopathy, and autonomic neuropathy, causing a functional loss in this organ. Thus, the hypercoagulatory state that is already part of the pathophysiology of COVID19 , is prone to an increase in its involvement, especially when associated with diabetic patients, causing severe damage to the body and contributing to unfavorable outcomes [12].

Hyperglycemia is affected by SARS-COV-2. The virus causes negative regulation of ACE-2 in the pâncreas[13] and higher levels of hyperglycemic hormones, such as glucocorticoids and catecholamines[14], causing decreased insulin secretion, increased resistance to insulin insulin and, consequently, secondary elevation of blood glucose. However, there is a possibility of direct damage to the pancreatic cells by the virus, with the presence of elevated levels of pancreatic amylase and lipase, and increased serum levels of fetuin A, a glycoprotein related to loss of sensitivity to insulin. Such factors corroborate for greater care with glycemic control and diabetes screening with the diagnosis of COVID-19, since the disease is silent and can be present after the worsening of the SARS-COV-2 infection, which impairs the prognosis [5].

The various mechanisms of the pathophysiology of diabetes also affect the immune system. Hyperglycemia has an immunosuppressive effect [15], impairing cell immunity, phagocytosis and chemotaxis [6], which will result in impaired dendritic cell function, with less interferon production $-\alpha$, malocyte malfunction, defects in complement action, reduced activation of natural killer cells (NK) and impaired innate immunity [16]. In contribution, SARS-COV-2 can attack lymphocytes, specifically $\mathrm{T}$ lymphocytes, which leads to lymphocytopenia [17]. Another reported finding was the increase in neutrophils in relation to lymphocytes and thrombocytopenia. Therefore, both humoral and cellular immunity are more prominent in the course of COVID-19 in diabetic patients, a fact that reveals a difficulty in controlling the infection and suggests the relationship with the higher prevalence of worsening in these patients [18].

\subsection{COVID-19 and Diabetes Medications}

Regarding the relationship between the medications routinely used for the treatment of diabetes mellitus and SARS-Cov-2 infection, 19 articles among those selected discussed the topic. Seven studies claim that oral antidiabetic agents such as pioglitazone (thiazolidinediones), liraglutide (glucagon-like peptide-1 agonist GLP-1), angiotensin-converting enzyme (ACE) inhibitors and angiotensin receptor blockers (BRA) can increase the risk of COVID-19 infection and more serious and fatal lung injuries.

On the other hand, two studies claim that there is still no evidence that the use of ACE or ARB enzyme inhibitors can affect the activity of the virus, nor the development of severe COVID-19 or greater risk of death.

Three studies indicate that metformin has beneficial effects in patients with diabetes with COVID-19. Suggesting that the use of metformin may be associated with a reduction in mortality.

Four studies claim that dipeptidyl peptidase 4 (DPP4I) inhibitors attenuated the severity of Covid-19. Two articles suggest that glibenclamide (sulfonylureas) can relieve lung injury. On insulin, its action provides a good essential glycemic control in cases of infection.

Antidiabetic agents, such as thiazolidinediones, GLP-1 agonists, ACE inhibitors, BRAs, increase the expression of ACE-2 [19]. Since SARS-CoV-2 uses ACE-2 as a receptor for entry into the host's pneumocytes, therefore, increased expression of ACE-2 would facilitate the entry and subsequent proliferation of the coronavirus. Since the virus uses the enzyme to enter the host tissue, ACE-2 has reduced expression and is unable to protect against lung injury [19].

However, despite the recognition of ECA-2 as the SARS CoV-2 receptor, and the role of ACE2 in lung injury; there are conflicting results with the use of ACEI and ARB in these patients [20]. Other studies have shown that human recombinant soluble ACE-2 significantly blocks SARS-CoV-2 infections, providing a justification that soluble ACE-2 can not only protect against lung injury, but also block the entry of SARS-CoV- 2 in the target cells[13].

Metformin may be useful in reducing oxidative stress and lung injury[13]. The results of observational studies show a reduction in mortality rates in patients treated with metformin while exposed to COVID-19[21]. 
Inhibitors of dipeptidyl peptidase 4 (IDPP4) attenuated lung injury and may have some antiinflammatory and antiproliferative effects on human lung microvascular endothelial cells cultured in vitro [13]. There is some data on the use of IDPP4 in previous viral epidemics. DPP4 is the main receptor for coronavirus of the Middle East respiratory syndrome (MERS), responsible for its entry into the cell. Therefore, the possibility of DPP4 facilitating the entry of SARS CoV-2 into the cell cannot be ruled out [20]. In this context, the role of DPP4 inhibition in the COVID-19 disease process needs to be explored [22].

Glibenclamide (sulfonylurea) can relieve acute lung injury by inhibiting the family of receptors similar to the nucleotide-binding oligomerization domain, the 3-pirin domain (NLRP3) of the inflammasome signaling pathway. In addition, glibenclamide disrupts pro-inflammatory cytokines and reactive oxygen species and suppresses the migration of inflammatory cells. However, the use of sulfonylureas in the hospital environment is discouraged due to the potential risk of hypoglycemia [13].

Insulin reduces the expression of ACE-2. In addition, the early administration of insulin in acute diseases is associated with better results and lower mortality rates, since hyperglycemia is commonly observed during acute and critical illnesses. Insulin inhibits the synthesis of proinflammatory factors, including TNF $\alpha$ and IL-6, and attenuates the oxidative stress observed in acute lung injury $[19,13]$.

\subsection{COVID-19 and Diabetes Management}

Of the 77 articles selected, 50 showed that the rigorous and effective control of DM is essentil for the adequate management of diabetic patients with COVID-19, especially with regard to plasma glucose levels. In this sense, 16 articles indicate the use of insulin as the therapy of choice for the control of hyperglycemia in non-critical hospitalized patients. In addition, there is evidence that it is safe to continue with the usual antidiabetic drugs in most patients with mild infection, good general condition and normal oral intake.

However, some medications such as sodium glucose-2 cotransporter inhibitors (SGLT-2) and metformin should be discontinued in dehydrated patients with severe forms of COVID-19. In addition, the use of sulphonylureas and glycnides should be discontinued due to the risk of hypoglycemia.
Although ACE-2 is suggested as an entry recipient of SARS-Cov 2 into the cell, the Brazilian Society of Diabetes and other international institutions recommend the maintenance of $\mathrm{ACE}$ or $\mathrm{ARB}$ medications to diabetic patients with COVID-19.

According to Pal and Bhadada (2020), the lack of control of the glycemic levels of the diabetic patient with COVID-19 compromises the innate immune response and promotes the generation of pro-inflammatory cytokines, establishing a vicious cycle [23]. Thus, the rigorous and effective management of DM in patients with COVID-19 is fundamental. In this sense, it is worth highlighting the role of insulin in the treatment of hyperglycemia in hospitalized patients, since it can prevent the rapid spread of inflammatory cytokine storms and lung injury in patients affected by the new coronavirus [24, 25]. Added to this, according to Nakhleh and Shehadeh (2020), insulin can restore ACE-2 and ADAM17 expression and RAS balance, as well as early administration to reduce the risk of developing diabetic ketoacidosis or hyperosmolar hyperglycemic states [13]. It is important to emphasize that SHANG et al. (2020) demonstrated that diabetic patients who need insulin present a higher risk of progression of the disease and worse prognosis after infection of SARS-Cov-2 [26].

Selvin and Juraschek (2020, p. 1692) propose routine care for diabetes patients, such as plasma and/or capillary blood glucose monitoring tests, routine tests recommended for DM complications, such as measuring urinary ketones, and glycated hemoglobin (Hba1c) tests [27]. In this context, Ceriello et al. (2020) recommend that the target blood glucose levels should be between 140-180 mg / dL for most patients [28].

There is evidence that it is safe to continue with the usual antidiabetic drugs in most patients with mild infection, good general condition and normal oral intake [12, 24, 11, 50]. Among these medications, there are LPG-1 analogues, which act in glycemic control and weight loss/maintenance, but diabetic patients with COVID-19 should be monitored due to dehydration, which can lead to the severity of the disease [30]. DPP-4 inhibitors, on the other hand, are generally quite tolerated and can be maintained. In addition, the study by Bassendine et al. (2020) pointed out that pro-inflammatory factors considerably increase the expression of DPP-4 in immune cells, parallel to this [22], Gupta, Hussain \& Misra (2020), suggest the 
possibility of DPP-4 to facilitate the entry of SARS-Cov-2 in the cell. Thus, the maintenance of DPP-4 inhibitors is relevant [24].

However, sodium glucose-2 cotransporter inhibitors (SGLT-2) should be disrupted by the risk of causing dehydration and diabetic ketoacidosis during the treatment of diabetic patients affected by the new coronavirus. In addition, metformin is recommended to be discontinued because of the risk of dehydration in patients with vomiting, low oral intake, concomitant sepsis, and severe impairment of liver and kidney function [8, 11, 31]. However, Scheen (2020) concluded that the use of metformin in the treatment of diabetic patients with COVID-19 may be associated with a reduction in mortality [21]. On the other hand, Nakhleh and Shehadeh (2020), cite that the use of sulfonylureas and glinidas should be discouraged in the hospital environment due to the potential risk of hypoglycemia [13].

ACE-2 appears to be the primary receptor for the entry of SARS Cov 2 into various epithelial tissues [32, 33]. Pititto and Ferreira point out that the angiotensin-converting enzyme 2 (ACE2 ) is increased in patients with DM 1 and 2 and that some medications may increase the expression of the ACE2 gene, such as ACE inhibitors (Iecas), angiotensin receptor blockers 2 (ARB)Thiazolidinediones and ibuprofen [34]. However, Filardi \& Morano (2020) concluded that the maintenance of ACE or ARB medications are not associated with increased risk of infection, severity or mortality due to COVID-19 [35]. The Brazilian Society of Diabetes, as well as other international institutions, do not recommend the suspension of these drugs due to SARS-Cov-2 infection [36]. In addition, Yang et al. (2020) recommend a healthy diet and physical activities to improve immunity [37].

\subsection{Diabetics Infected with SARS-COV-2: Prognosis}

50 of the researched articles mention that diabetic status is indicative of a worse prognosis, with chances of complications. Thus, statistically, diabetic patients are more likely to require admission to Intensive Care Units (ICUs), in addition to presenting worse morbidity and mortality results when affected by pulmonary infections. However, it is not entirely clear, among the authors, the mechanism by which the hyperglycemic state contributes to the probability of a more negative outcome for COVID-19. It is also noteworthy that other chronic diseases, such as systemic arterial hypertension and chronic lung diseases, are scored as co-participants of the worst prognosis. Furthermore, it is not yet determined whether the prognosis for progression to severe forms of COVID is the same among all types of DM.

Regarding the prognosis of COVID-19, since the beginning of the pandemic, it is known that diabetes melitus (DM) is one of the high risk factors for the development of unfavorable results [38]. Besides that, Papadokostaki, Tentolouris and Liberopoulos (2020) clarify that in diabetic patients with overlapping obesity, the use of mechanical ventilation is required, in a greater number of times [12].

Thus, it is suggested that the exacerbation of the inflammatory state, which occurs in diabetic patients, contributes to the concomitant SARSCOV-2 infection being associated with an increased risk of complications, such as pulmonary infections. However, the mechanism by which the hyperglycemic state aggravates the condition of patients infected with the novel coronavirus is not yet fully understood $[39,6]$.

In this context, Scott, Jenkins and Fulcher (2020) cite that, in a meta-analysis of 6 studies, which evaluated 1527 people, it was possible to notice that diabetic patients infected with SARS-COV-2 complicate in $11.7 \%$ of cases [40]. Nevertheless, in non-diabetic patients with COVID-19, the observed complication rate is much lower, being around 4\%. Moreover, the Ministry of Health emphasizes that most cases of death occur in patients with some pre-existing comorbidity, such as: hypertension, diabetes, cardiovascular disease, cerebrovascular disease, chronic lung disease, cancer and others [41].

In addition, Yang et al 2020 compared the mortality of COVID-19 between diabetics and non-diabetics, and affirm that among diabetics, those with DM-2 have an increased incidence of morbidity, when compared to those with DM-1 and those with special forms of DM [38]. Pal and Bhadada (2020) mention, however, that no clear distinction was made between type 1 diabetes mellitus and type 2 diabetes mellitus, and it is likely that both types of DM are predictors of poor prognosis regarding COVID19 [23].

Parallel to this, it should be noted that not all authors considered diabetes as a risk factor for the development of severe Covid-19. Thus, against what most of the analyzed articles concluded, for Baradaram et al, authors of the 
systematic review entitled "Prevalence of Comorbidities in COVID-19 Patients: A Systematic Review and Meta-Analysis", which aimed to assess the prevalence of comorbidities in patients with confirmed COVID-19, only hypertension is responsible for the increase in mortality.

In this sense, according to this article, the risk of complications is primarily associated with age. Therefore, since people of older age usually have some associated disease, comorbidities end up being seen in the epidemiology of the new coronavirus. Therefore, with the exception of hypertension, the existence of chronic diseases would not be a prerequisite for the development of the severe form of COVID. It should be noted, however, that the study by Baradaram et al (2020) was limited to the investigation of 33 articles, in China and Taiwan, only on April 7, 2020 [42].

Further, another important aspect to be mentioned is that DM and COVID-19 interact mutually. Therefore, not only does DM cause worsening of those infected with SARS-COV-2: infection by COVID-19 is also capable of causing complications to the diabetic state. Thus, it is noted that infection with the novel coronavirus makes glycemic control in diabetic individuals more difficult than other critical conditions are capable of generating [23].

Accordingly, good glycemic control helps to reduce the risk of complications in patients infected with SARS-COV-2, since the hyperglycemic state can contribute to the increase in virulence of certain pathogens. Thus, it is mandatory that patients are monitored with frequent blood glucose monitoring, aiming at a glycemic target between $100-180 \mathrm{mg} / \mathrm{dl}$ [10].

Besides, Gosh, Gupta and Misra (2020) mention telemedicine as an important ally in the health care of diabetic patients, during the pandemic, since it allows the monitoring of diabetic patients to be followed up, without having to leave the house [43].

\section{CONCLUSiOnS}

The endocrine associations between diabetes mellitus and COVID-19 demonstrate an important relationship in their pathophysiology. $\mathrm{DM}$ is one of the risk factors for the development of unfavorable results. In addition, it is important to note that in cases of SARSCov-2 infection, hypoglycemic medications should not be discontinued without clinical indication, and good glycemic control in these patients is essential.

\section{CONFLICT OF INTEREST}

None

\section{FUNDING SOURCE}

None

\section{REFERENCES}

[1] International Diabetes Federation. IDF Diabetes Atlas, 8 Edition. www.idf.org/elibrary/epidemiology-research/diabetesatlas/134-idf-diabetes-atlas-8th-edition.html. Acessed 4 Sep 2020.

[2] Jeong IK, Yoon KH, Lee MK. Diabetes and COVID-19: Global and regional perspectives. Diabetes Research and Clinical Practice. 2020; 166: 108303 .

[3] Sandooja R, Vura NVRK, Morocco M. Heightened ACE Activity and Unfavorable Consequences in COVID-19 Diabetic Subjects. International Journal of Endocrinology. 2020.

[4] Huang I, Lim MA, Pranata R. Diabetes mellitus is associated with increased mortality and severity of disease in COVID-19 pneumonia A systematic review, meta-analysis, and metaregression. Diab \& Metab Syndr: Clin Res \& Ver. 2020; 14(4): 395-403. Available on: https://doi.org/10.1016/j.dsx.2020.04.018.

Accessed in August 2020.

[5] Chee YJ, Tan SK, Yeoh E. Dissecting the interaction between COVID-19 and diabetes mellitus. Journal of Diabetes Investigation. 2020; 1-11.

[6] Barber T. COVID-19 and diabetes mellitus: implications for prognosis and clinical management. Journal Expert Review of Endocrinology \&amp; Metabolism. 2020; 15(4): 227-236. Available on: https://www.tandfonline.com/doi/full/10

.1080/17446651.2020.1774360. Accessed August 2020.

[7] Pal R, Banerjee M. COVID-19 and the endocrine system: exploring the unexplored. J Endoc Investig. 2020; 43: 1027-1031. Available on: https://doi.org/10.1007/s40618020-01276-8.0. Accessed August 2020.

[8] Mazucanti CH, Egan JM. SARS-CoV-2 disease severity and diabetes: why the connection and what is to be done?. Immun Ageing. 2020; 17(21): 1-11. Available on: https://doi.org/10.1186/s12979-020-00192-y.

Accessed August 2020.

[9] Azar W, Njeim R, Fares A, Azar N, Azar S, Sayed $M$ et al. COVID-19 and diabetes mellitus: how one pandemic worsens the other. Reviews in Endocrine and Metabolic Disorders, 2020. 
[10] Korytkowski M, Antinori-Lent K, Drincic A, Hirsch I, McDonnel M, Rushakoff R et al. A Pragmatic Approach to Inpatient Diabetes Management during the COVID-19 Pandemic. The Journal of Clinical Endocrinology \& Metabolism. 2020; 105 (9): 3076-3087. Available on: https://academic.oup.com/jcem/article/105/9/30 76/5851514. Accessed August 2020.

[11] Bornstein S, Rubino F, Khunti K, Mingrone G, Hopkins D, Birkenfeld AL et al. Practical recommendations for the management of diabetes in patients with COVID-19. The Lancet. $\quad 2020 ; \quad 8 \quad$ (6):546-550. Doi:10.1016/S2213-8587(20)30152-2.

Available on: https://www.thelancet.com/journals/landia/artic le/PIIS2213-8587(20)30152-2/fulltext. Accessed August 2020.

[12] Papadokostaki E, Tentolouris N, Liberopoulos E. COVID-19 and diabetes: What does the clinician need to know? Primary care diabetes. 2020; 14 (5): 558-563. Available on: https://doi.org/10.1016/j.pcd.2020.06.010. Accessed August 2020.

[13] Nakhleh A, Shehadeh N. Interactions between antihyperglycemic drugs and the reninangiotensin system: Putative roles in COVID19. A mini-review. Diab \& Metab Syndr: Clin Res \& Rev. 2020; 14(4):509-512. Available on: https://doi.org/10.1016/j.dsx.2020.04.040. Accessed August 2020.

[14] Hussain A, Bhowmik, B, Moreira, NCV. COVID-19 and diabetes Knowledge in progress.Diabetes Research and Clinical Practice. 2020. 162. 10.1016 / j.diabres.2020.108142.

[15] Rod JE, Oviedo-Trespalacios O, CortesRamirez J. A brief-review of the risk factors for covid-19 severity. Rev. Saúde Pública. 2020; 54(60).Available on: http://www.scielo.br/scielo.php?script=sci_artte xt\&pid=S0034-

$89102020000100701 \& \operatorname{lng}=\mathrm{en} \& \mathrm{nrm}=\mathrm{iso}$. Accessed August 2020.

[16] Grupta R, Hussain A, Misra A. Diabetes and COVID-19: evidence, current status and unanswered research questions. Eur J Clin Nutr. 2020; 74:864-870 864-870 Available on:https://www.nature.com/articles/s41430020-0652-1\#citeas. Accessed August 2020.

[17] El-Missiry MA, El-Missiry, ZMA, Othman AI. Melatonin is a potential adjuvant to improve clinical outcomes in individuals with obesity and diabetes with coexistence of Covid-19. European Journal of Pharmacology. 2020; 882: 173329.
[18] Salib C, Tfeldstein J. Hypersegmented granulocytes and COVID-19 infection. Blood. 2020; 135 (24):2196.

[19] Dariya B, Nagaraju, GP. Understanding novel COVID-19: Its impact on organ failure and risk assessment for diabetic and cancer patients. Cytokine \& Growth Factor Reviews. 2020; 53: 43-52. 43-52. Available on:https://doi.org/10.1016/j.cytogfr.2020.05.00 1. Accessed August 2020.

[20] Gupta R., Misra A.Contentious issues and evolving concepts in the clinical presentation and management of patients with COVID-19 infectionwith reference to use of therapeutic and other drugs used in Co-morbid diseases (Hypertension, diabetes etc).Diabetes \& Metabolic Syndrome: Clinical Research \& Reviews. 2020; 14(3):251-254. Available on: https://pubmed.ncbi.nlm.nih.gov/32247213/. Accessed August 2020.

[21] Schenn A. Metformin and COVID-19: From cellular mechanisms to reduced mortality. Diabetes \& Metabolism. 2020. Available on: https://www.sciencedirect.com/science/artic le/pii/S1262363620300987?via\%3Dihub. Acce ssed August 2020.

[22] Bassendine MF, Bridge SH, McCaughan GW, Gorrell MD. COVID-19 and comorbidities: A role for dipeptidyl peptidase 4 (DPP4) in disease severity?. J Diabetes. 2020; 12 (9): 649$658 . \quad$ Available on: https://onlinelibrary.wiley.com/doi/full/10.1111 /1753-0407.13052. Accessed August 2020.

[23] 23.Pal R , Bhadada, SK. COVID-19 and diabetes mellitus: An unholy interaction of two pandemics. Diab \& Metab Syndr: Clin Res \& Rev. 2020; 14 (4):513-517. Available on: https://doi.org/10.1016/j.dsx.2020.04.049. Accessed in August 2020.

[24] Gupta R, Ghosh A, Singh AK, Misra A. Clinical considerations for patients with diabetes in times of COVID-19 epidemic. Diab Metab Syndr.: Clin Res \& Ver. 2020; 14(3):211-212. Available on: https://doi.org/10.1016/j.dsx.2020.03.002. Acce ssed in August 2020.

[25] Kakoulidis I, Ilias I, koukkou E. SARS-CoV-2 infection and glucose homeostasis in pregnancy. What about antenatal corticosteroids?. Diab \& Metab Syndr: Clin Res \& Rev. 2020; 14(4):519-520. Available on: https://doi.org/10.1016/j.dsx.2020.04.045. Acce ssed August 2020.

[26] Shang J, Wang Q, Zhang H, Wang X, Wan J, Yan $Y$ et. al. The Relationship Between Diabetes Mellitus and COVID-19 Prognosis: A Retrospective Cohort Study in Wuhan, China. The American Journal of Medicine. 2020. Available

on: 
https://www.amjmed.com/article/S00029343(20)30532-5/fulltext. Accessed August 2020.

[27] Selvin E, Juraschek SP. Diabetes Epidemiology in the COVID-19. Diabetes Care. 2020; 43(8):1690-1694. Available on: https://care.diabetesjournals.org/content/43 /8/1690. Accessed August 2020.

[28] Ceriello A, Standl E, Catrinoiu D, Lalic N, Schnell O, Valensi $\mathrm{P}$ et al. Issues for the management of people with diabetes and COVID-19 in ICU. Cardiovascular diabetology. 2020; 19(1):114. Available on: https://doi.org/10.1186/s12933-020-01089-2. Accessed August 2020.

[29] Gosh A., Gupta R., Misra A. Telemedicine for diabetes care in India during COVID19 pandemic and national lockdown period: Guidelines for physicians. Diabetes \& Metabolic Syndrome: Clinical Research \& Reviews, 2020; 14(4):273-276. Available on: https://pubmed.ncbi.nlm.nih.gov/32283497.Acc essed August 2020.

[30] Silva LED, Cohen RV, Andrade JC, Szego T, Santo MA, Ramos AC et al. The recommendations of the Brazilian College of Surgeons and the Brazilian Bariatric and Metabolic Surgery Societies on the return of bariatric and metabolic operations in geographic regions of the country where the procedures have been allowed by local policies, in the period of COVID-19 pandemic. Rev. Col. Bras. Cir. 2020; 47. Available on: http://www.scielo.br/scielo.php?script=sci_artte $\mathrm{xt} \& \mathrm{pid}=\mathrm{S} 0100$ -

69912020000100606\&lng=en\&nrm=iso.

Accessed August 2020.

[31] Wake DJ, Gibb FW, Kar P, Kennon B, Klonoff DC, Rayman G et al.Endocrinology in the time of COVID-19: Remodelling diabetes services and emerging innovation. Eur J Endocr. 2020; 183(2):67-77. Available on: https://doi.org/10.1530/EJE-20-0377. Accessed August 2020.

[32] Brufsky A. Hyperglycemia, hydroxychloroquine, and the COVID-19 pandemic. J Med Virol. 2020; 92(7): 770775. Available

on: https://doi.org/10.1002/jmv.25887. Accessed August 2020.

[33] Sławiński G, Lewicka E. What should a cardiologist know about coronavirus disease 2019?. Kardiol Pol. 2020; 78:278283. Available on: https://doi.org/10.33963/KP.15302. Accessed August 2020.

[34] Pititto BA, Ferreira SRG. Diabetes and covid19: more than the sum of two morbidities. Rev. Saúde Pública. 2020; 54. Available on: http://www.scielo.br/scielo.php?script=sci_artte xt\&pid=S0034-89102020000100603\&lng=en \&nrm=iso. Accessed August 2020.

[35] Filardi T, Morano S. COVID-19: is there a link between the course of infection and pharmacological agents in diabetes?, J Endocrinol Invest. 2020; 43(8):10531060.Available

on:https:/www.ncbi.nlm.nih.gov/pmc/articles/ PMC7268955/\#!po=55.7692. Accessed August 2020.

[36] Brazilian Society of Diabetes. Note on angiotensin converting enzyme (ACEI) inhibitors, angiotensin receptor blockers (BRA) and COVID-19. Rio de Janeiro:BSD. 2020. Available on: https://www.diabetes.org.br/comunicados/noteon-angiotensin-converting-enzyme-inhibitorsangiotensin-receptor blockers-and-covid-19/. Accessed August 2020.

[37] Yang YMM, Wen Z, Yuan T, Chunguang X, Xiauoxu F, Hui Z. The effect of diabetes on mortality of COVID-19. Medicine. 2020; 99(27). Available on: https://journals.lww.com/mdjournal/FullText/2020/07020/The_effect_of_di abetes_on_mortality_of_COVID_19_A.65.asp x. Accessed August 2020.

[38] Cristelo C, Azevedo C, Marques JM, Nunes R, Sarmento B et al. SARS-CoV-2 and diabetes: New challenges for the disease. Diabetes Research and Clinical Practice. 2020; 164. Available on: https://www.sciencedirect.com/science/article/p ii/S0168822720304782. Accessed August 2020.

[39] Hu Y, Sun J, Dai Z. et. al. Prevalence and severity of coronavirus disease 2019 (COVID19): A systematic review and meta-analysis. J Clin Virol. 2020; 127. Available on: https://doi.org/10.1016/j.jcv.2020.104371. Accessed August 2020.

[40] Scott ES, Jenkins AJ, Fulcher GR. Challenges of diabetes management during the COVID-19 pandemic. Med. J. Aust. 2020; 213(1):56-57. Available

on:https://www.mja.com.au/journal/2020/challe nges-diabetes-management-during-covid-19pandemic?utm_source $=$ carous el\&utm_medium=web\&utm_campaign=homep age. Accessed August 2020.

[41] Brazil. Ministry of Health. Health Surveillance Secretariat. Care for People with Chronic Diseases in PHC Facing the Pandemic Situation of COVID-19. 2020. Available on:http://fiadmin.bvsalud.org/document/view/m6yu5. Accessed August 2020. 
[42] Baradaran A., Ebrahimzadeh M.H., Kachooei A.R. Prevalence of Comorbidities in COVID19 Patients: A Systematic Review and MetaAnalysis.The Archives of Bone and Joint Surgery. 2020; 8:247-255. Available on: https://www.ncbi.nlm.nih.gov/pmc/articles/PM C7296605/. Accessed August 2020.
[43] Gosh A, Gupta R, Misra A. Telemedicine for diabetes care in India during COVID19 pandemic and national lockdown period: Guidelines for physicians. Diab \& Metab Syndr: Clin Res \& Rev. 2020; 14: 273-276. Available on: https://pubmed.ncbi.nlm.nih.gov/ 32283497/. Accessed August 2020.

Citation: Wallace Rodrigues de Holanda Miranda et al. Covid-19 and Diabetes Mellitus: What do you know?. ARC Journal of Diabetes and Endocrinology. 2020; 6(1):22-30. DOI: https://doi.org/10.20431/2455-5983.0601004.

Copyright: (C) 2020 Authors. This is an open-access article distributed under the terms of the Creative Commons Attribution License, which permits unrestricted use, distribution, and reproduction in any medium, provided the original author and source are credited. 